\title{
Core requirements of the adenine riboswitch aptamer for ligand binding
}

\author{
JEAN-FRANÇOIS LEMAY and DANIEL A. LAFONTAINE \\ Département de biologie, Faculté des sciences, Université de Sherbrooke, Québec, J1K 2R1, Canada
}

\begin{abstract}
The adenine riboswitch aptamer, the $\mathrm{A}$ box, positively regulates gene expression upon adenine binding. To provide insight into structure-function relationships, important for the adenine riboswitch aptamer, we have created alignments for six aptamer sequences that reveal the core requirements. In addition, 2-aminopurine (2AP) binding studies have been used to test the consensus sequence derived from the alignment. Overall, the consensus secondary structure is consistent with 2AP binding studies. However, a position in the core, previously identified as variable, shows restriction in nucleotide sequence. Furthermore, this restriction is found to be related with the ligand specificity of the riboswitch. The implications of this relationship for the riboswitch gene regulation mechanism are discussed.
\end{abstract}

Keywords: riboswitch; RNA structure; RNA folding; aptamer; molecular recognition; fluorescence spectroscopy

\section{INTRODUCTION}

Riboswitches are regulatory elements found predominantly in 5'-untranslated regions of messenger RNAs (mRNAs). These genetic switches are highly structured domains that can bind cellular metabolites and regulate the expression of proximally located downstream gene sequences without the presence of protein cofactors (for review, see Nudler and Mironov 2004; Soukup and Soukup 2004; Tucker and Breaker 2005; Winkler 2005; Winkler and Breaker 2005). Riboswitches are composed of two structural domains: a ligand binding aptamer, and an expression platform. The aptamer exhibits a high degree of sequence and structural conservation and is specific for a given metabolite. Expression platforms vary widely in sequence and structure, and typically affect gene expression through formation of Rho-independent transcriptional terminators or through sequestration of the Shine-Dalgarno sequences required for translation initiation (Soukup and Soukup 2004). Nine classes of bacterial riboswitches are known and respond to adenine (Mandal and Breaker 2004a), adenosylcobalamin (Nahvi et al. 2002, 2004), flavin mononucleotide (Mironov et al. 2002; Winkler et al. 2002b), guanine (Mandal et al.

Reprint requests to: Daniel A. LaFontaine, Département de biologie, Faculté des sciences, Université de Sherbrooke, Québec, J1K 2R1, Canada; e-mail: Daniel.Lafontaine@Usherbrooke.ca; fax: (819) 821-8049.

Article published online ahead of print. Article and publication date are at http://www.rnajournal.org/cgi/doi/10.1261/rna.142007.
2003), glucosamine-6-phosphate (Winkler et al. 2004), glycine (Mandal et al. 2004), lysine (Grundy et al. 2003; Sudarsan et al. 2003b), intracellular magnesium (Cromie et al. 2006), S-adenosylmethionine (Epshtein et al. 2003; McDaniel et al. 2003; Winkler et al. 2003), and thiamine pyrophosphate (Mironov et al. 2002; Winkler et al. 2002a). In addition, it has been suggested that riboswitches can directly control mRNA splicing (Kubodera et al. 2003; Sudarsan et al. 2003a) and stability (Winkler et al. 2004) in higher organisms.

Purine-specific riboswitches comprise the adenine and the guanine riboswitch classes, which are among the smallest riboswitches known to date. Despite the high structural similarity shown between these aptamers, they display very high specificity, discrimination, and affinity toward their respective ligands, adenine and guanine (Mandal et al. 2003; Mandal and Breaker 2004a). Although the guanine riboswitch regulation appears to be strictly by attenuating transcription (Mandal et al. 2003), the adenine riboswitch positively regulates gene expression (Mandal and Breaker 2004a) by modulating the structure of a transcription terminator (Fig. 1) and also by modulating sequences that sequester the Shine-Dalgarno and initiation codon sequences (Serganov et al. 2004). Both purine aptamers are predicted to fold as a three-way junction where the P1 stem is the only helical region showing some degree of conservation, presumably to interact with the expression platform (Mandal et al. 2003; Mandal and Breaker 2004a), 

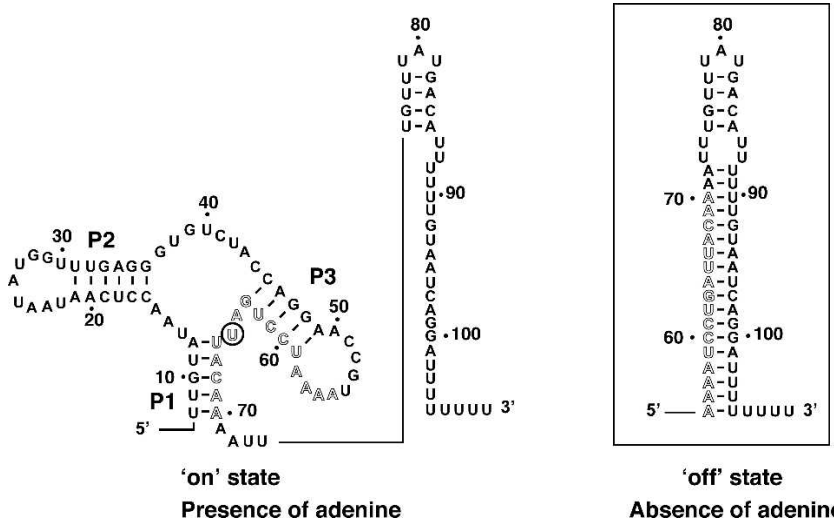

FIGURE 1. Secondary structure models for the $p b u E$ adenine riboswitch. Alternative base pairing of the riboswitch associated with "off" and "on" states. The on state is characterized by the presence of the aptamer, while the off state is characterized by the presence of a transcription terminator stem (boxed). Only the terminator stem is shown in the off state. The aptamer is expected to be stabilized by binding of adenine. U65 is circled as it is the nucleotide that determines ligand specificity. Outlined letters represent nucleotides that are involved in the formation of both the terminator and the aptamer structures.

while helices P2 and P3 are not conserved but are involved in a loop-loop interaction (Batey et al. 2004; Serganov et al. 2004; Gilbert et al. 2006). Eight riboswitch aptamer structures have been determined by X-ray crystallography (Batey et al. 2004; Serganov et al. 2004; Edwards and FerreD'Amare 2006; Gilbert et al. 2006; Klein and FerreD'Amare 2006; Montange and Batey 2006; Serganov et al. 2006; Thore et al. 2006). In each complex, the fold of the RNA fragment is very compact, with many intricate tertiary interactions (Lescoute and Westhof 2005). Crystal structures show that the bound ligand is directly involved in a Watson-Crick base pair with the nucleotide at position 65 (Batey et al. 2004; Serganov et al. 2004; Lescoute and Westhof 2005; Gilbert et al. 2006), making this residue particularly important, since conversion of an adenine-sensing to a guanine-sensing riboswitch has been achieved by replacing the uracil at position 65 by a cytosine (Mandal and Breaker 2004a).

Consensus sequences and secondary structures have been reported for the adenine-specific (A box) (Breaker 2004; Soukup and Soukup 2004) and the guanine-specific (G box) (Mandal et al. 2003; Soukup and Soukup 2004) aptamers. Given their very similar primary and secondary structures, a purine consensus secondary structure has emerged where high sequence conservation occurs within the unpaired regions found in the loops and in the core of the aptamer (Winkler and Breaker 2003; Bengert and Dandekar 2004; Mandal and Breaker 2004b; Winkler and Breaker 2005). A structural ground is provided for the sequence conservation by in-line probing experiments where the core of the aptamer has been found to undergo a major structural reorganization upon ligand binding
(Mandal et al. 2003; Mandal and Breaker 2004a). Here, using 2-aminopurine (2AP) as a fluorescent ligand to monitor the formation of an aptamer-ligand complex, we study the core requirements of the adenine-sensing aptamer for ligand binding. Although the adenine aptamer is highly similar to that of the guanine, we find that the adenine aptamer has different sequence requirements in the core region. Because these differences are directly related to the ligand specificity of the riboswitch, we conclude that both adenine and guanine aptamers have different core requirements that are adapted for the particular riboswitch ligand specificity.

\section{RESULTS}

\section{Sequence and secondary structure consensus of the A box motif}

Three representatives of the adenine riboswitch have originally been reported (Mandal et al. 2003). One variant is located upstream of the $p b u E$ gene (Fig. 1) that encodes a purine efflux pump of Bacillus subtilis (Johansen et al. 2003; Nygaard and Saxild 2005), while the other two are found to regulate an adenosine deaminase gene $(a d d)$ in Vibrio vulnificus and Clostridium perfringens. An additional sequence has also been found upstream of an add gene from Vibrio parahaemolyticus using a dedicated RNA motif search program (Bengert and Dandekar 2004). The previous discovery encouraged us in an attempt to identify new adenine riboswitch representatives in bacterial genomes. We reasoned that having a larger subset of adenine riboswitch sequences should help us to define a more accurate A box consensus sequence and therefore a better understanding of the core requirements for this aptamer. Using the BLAST engine (Altschul et al. 1990), we found two novel adenine riboswitch sequences in Colwellia psychrerythraea and Photobacterium profundum that are both located upstream of an adenosine deaminase gene. Thus, by using a comparative sequence strategy with all six variant sequences, we identified several conserved sequence elements that have been described in previous studies (Fig. 2A; Mandal et al. 2003; Mandal and Breaker 2004a). From this alignment, two differences in sequence conservation are observed in the core when compared to the purine consensus (Fig. 2B; Mandal and Breaker 2004a). One is located at position 15 where a strict requirement for an adenine is found in our alignment, while this position is nonconserved in the purine consensus sequence. Notably, a recent review by Breaker has reported an A box consensus sequence, and has also indicated a strict necessity for an adenine at this position (Breaker 2004). The other difference is found at position 39. This position has been previously identified as completely nonconserved ("N" sequence consensus) (Breaker 2004). However, our alignment predicts a different consensus where all nucleotides 
A
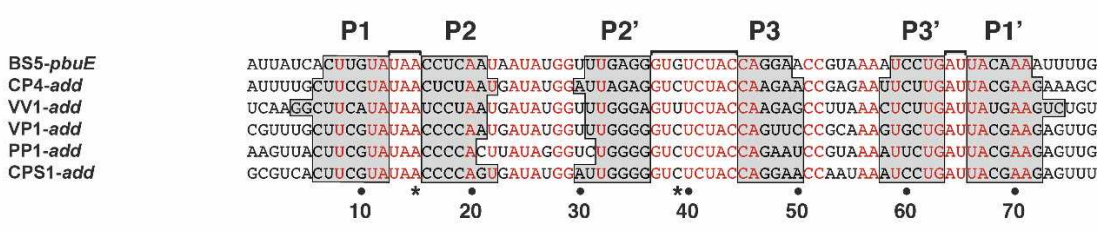

B

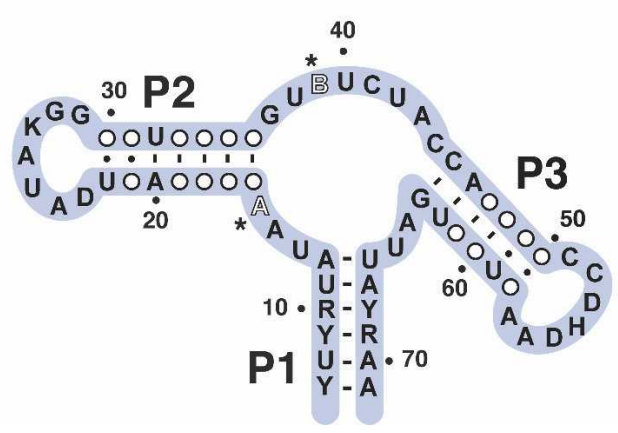

FIGURE 2. The adenine riboswitch aptamer structure and sequence consensus. (A) Sequence alignment of six representative mRNA domains that conform to the adenine riboswitch aptamer motif. Shaded regions represent helical regions P1, P2, and P3. Nucleotides in red are conserved throughout the entire alignment. Horizontal brackets indicate single-stranded regions that form the core of the aptamer. Gene names are as annotated as previously reported (Mandal et al. 2003). Organisms abbreviations are as follows: Bacillus subtilis (BS), Clostridium perfringens (CP), Colwellia psychrerythraea (CPS), Photobacterium profundum (PP), Vibrio parahaemolyticus (VP), and Vibrio vulnificus (VV). Asterisks identify the two nucleotides that are studied in this work. $(B)$ The consensus sequence and secondary structure for the A box motif. R and $\mathrm{Y}$ denote purine and pyrimidine, respectively. B, D, H, and $\mathrm{K}$ stand for $\mathrm{G} / \mathrm{U} / \mathrm{C}, \mathrm{G} / \mathrm{U} / \mathrm{A}, \mathrm{A} / \mathrm{U} / \mathrm{C}$, and $\mathrm{G} / \mathrm{U}$, respectively. Circles represent Watson-Crick base pairs that are not strongly conserved in helices P2 and P3. Asterisks identify the two nucleotides that are studied in this work.

are allowed except an adenine ("B" sequence consensus). Given that ligand binding occurs in the core of the aptamer, we targeted our analysis to this region.

\section{AP fluorescence is efficiently quenched by adenine-specific aptamers}

The ligand binding affinity for newly found adenine riboswitch aptamers was studied using the fluorescent ligand 2AP. Formation of a complex between $2 \mathrm{AP}$ and the $p b u E$ adenine-sensing aptamer has previously been studied by us (Lemay et al. 2006) and others (Wickiser et al. 2005; Gilbert et al. 2006), where it was shown that $2 \mathrm{AP}$ fluorescence becomes significantly quenched upon binding of the aptamer. Our work has also shown that 2AP fluorescence quenching can be used to determine the affinity of the complex, and an apparent dissociation constant $\left(K_{\text {Dapp }}\right)$ of $354 \mathrm{nM}$ has been determined, which is in excellent agreement with in-line probing studies (Mandal and Breaker 2004a). 2AP fluorescence quenching is consistent with the crystallographic structure of the A box-adenine complex, where the bound adenine is completely surrounded by RNA, both along its periphery and with base stacking on both sides of the ligand (Serganov et al. 2004). A representative example is shown in Figure 3A, where complex formation is monitored as a function of the RNA concentration of the VV1 adenine riboswitch aptamer. In absence of the aptamer, a relatively high fluorescence signal is observed (Fig. 3A, inset). As the concentration of the aptamer is increased, the $2 \mathrm{AP}$ fluorescence intensity progressively declines until becoming almost totally quenched in the presence of $2 \mu \mathrm{M}$ aptamer. The fluorescence data were fitted to a simple two-state binding model (see Materials and Methods). An apparent dissociation constant value of $116 \pm 14 \mathrm{nM}$ was obtained for the VV1 aptamer molecule. The 2AP binding assay was used to determine the ligand binding affinity for each adenine aptamer included in our alignment, and all calculated values are indicated in Table 1. Relative affinities $\left(K_{\mathrm{D}}{ }^{\mathrm{rel}}\right)$ reported to the pbuE aptamer are also indicated as a comparison and range from 0.3 (VV1 aptamer) to 1.6 (CP4 aptamer), making VV1 the aptamer having the best affinity for 2AP. Thus, all six variants exhibit efficient $2 \mathrm{AP}$ binding and can be confidently used in the generation of the reported A box sequence alignment and consensus secondary structure (Fig. 2).

In addition, we tested whether a natural $G$ box from the $x p t-p b u X$ operon (Mandal et al. 2003) was able to quench 2AP fluorescence. A titration of the $G$ box aptamer (Fig. 3B) was performed in the presence of $2 \mathrm{AP}$ and a $K_{\text {Dapp }}$ of $3747 \mathrm{nM}$ was obtained. This corresponds to an affinity decrease of 11-fold when compared to the pbuE A box aptamer (Table 1). Switching the specificity of guanine and adenine aptamers has previously been achieved by performing a single $\mathrm{U}$ to $\mathrm{C}$ substitution at position 65 (Mandal and Breaker 2004a). To assess if 2AP exhibits the same specificity as adenine toward position 65 , a construct was engineered in which a $\mathrm{C} 65 \mathrm{U}$ mutation was introduced in the context of the G box. The 2AP fluorescence quenching assay yielded a $K_{\text {Dapp }}$ of $390 \mathrm{nM}$ corresponding to a $K_{\mathrm{D}}{ }^{\text {rel }}$ of 1.1 , which is very close to the wild-type A box binding affinity (Table 1). Thus, these results show that 2AP binding is more efficient with aptamers having a uracil at position 65 corresponding to adenine-specific aptamers.

\section{Importance of base-paired regions for 2AP binding}

According to the adenine riboswitch aptamer consensus secondary structure (Fig. 2B) and to a recently described 
A

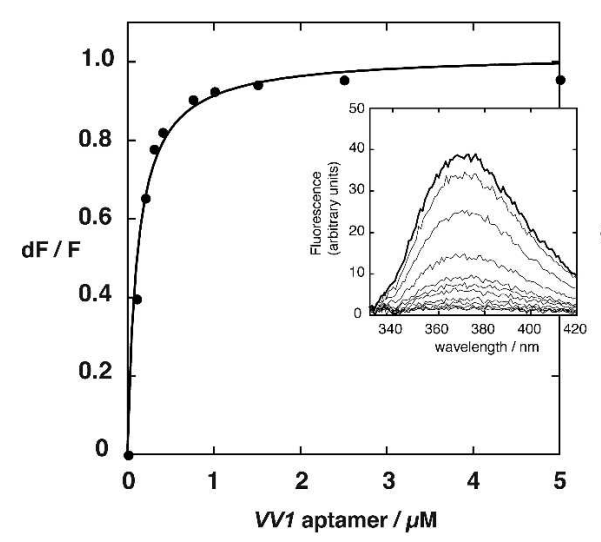

B

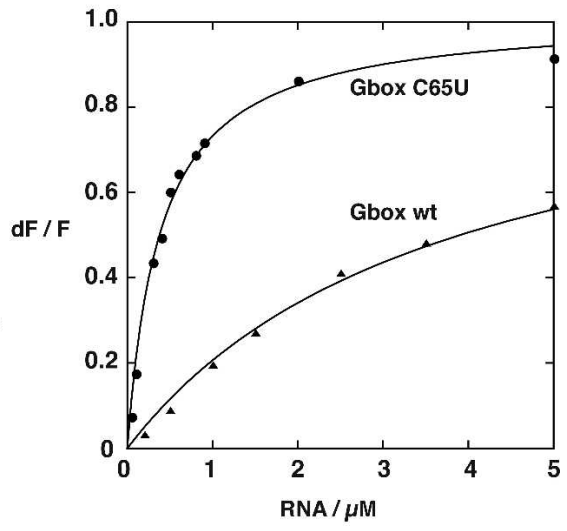

C

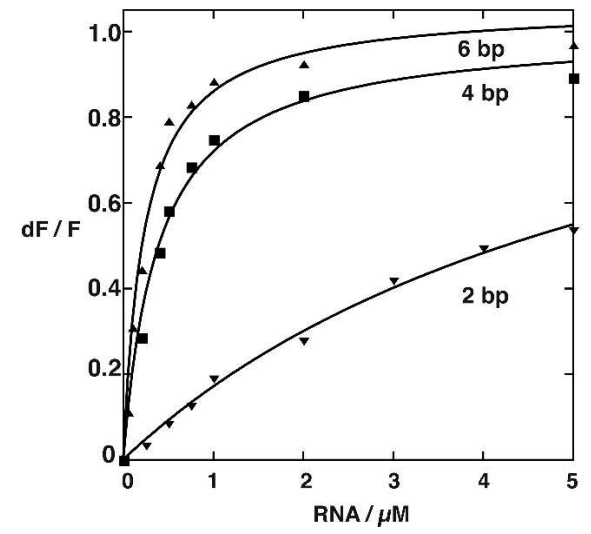

FIGURE 3. Purine aptamers quench 2AP fluorescence with a different affinity. (A) Normalized 2AP fluorescence intensity plotted as a function of VV1 A box RNA concentration. Changes in fluorescence $(\mathrm{dF})$ were normalized to the maximum fluorescence measured in the absence of A box RNA (F). The line shows the best fit to a simple binding model (see Materials and Methods), yielding a $K_{\text {Dapp }}$ of $116 \pm 14 \mathrm{nM}$. The inset shows fluorescence emission spectra $\left(\lambda_{\mathrm{ex}}=300 \mathrm{~nm}\right)$ from 330 to $420 \mathrm{~nm}$ for each RNA concentration. The bold line represents the $2 \mathrm{AP}$ fluorescence spectrum in the absence of RNA. The fluorescence intensity decreases with each addition of RNA. $(B)$ The $x p t-p b u X$ G box binds $2 \mathrm{AP}$ with a reduced affinity. Conversion to a adenine-sensing $\mathrm{G}$ box by the introduction of a C65U mutation increases 2AP binding affinity. (C) P1 stem length is important for 2AP binding. This analysis employed a series of variant $V V 1$ aptamers comprising an elongated helix P1 that varied in length from 2 to $6 \mathrm{bp}$.

consensus A box structure (Breaker 2004; Soukup and Soukup 2004), most of the base-paired regions are not highly conserved. However, the P1 stem and the P3 junctionproximal base pairs (bp) (C45-G63 and A46-U62) are conserved to some degree. To test if this evolutionary conservation is a requirement for ligand binding, we introduced mutations at these positions and resulting molecules were tested for 2AP binding. A first mutation, C45G-G63C, was introduced in the $\mathrm{A}$ box aptamer and was assayed for 2AP fluorescence binding (Table 2). An 8.2-fold decrease in $2 \mathrm{AP}$ binding was observed indicating that the identity of the C45-G63 base pair has a significant importance for ligand binding. The identity of the adjacent base pair A46-U62 was also tested and a $K_{\mathrm{D}}{ }^{\text {rel }}$ value of 3.5 was obtained, showing that the identity of this base pair is also important for $2 \mathrm{AP}$ binding (Table 2). However, the nature of two base pairs, A20-U32 and A49U59, are not important for 2AP binding given that $K_{\mathrm{D}}{ }^{\text {rel }}$ values close to unity are obtained when these base pairs are mutated (Table 2).

The P1 stem is a key element in the adenine-regulated riboswitch mechanism because its formation directly modulates the equilibrium between the "on" and "off" conformer structures (Fig. 1). This region was also tested, as it is the only helical segment of the A box aptamer showing an evolutionary conservation. A first variant having the base pair A12-U66 was examined by introducing an A12U-U66A mutation. The binding affinity of this mutant is reduced by a factor of 3.8 , showing the relative importance of the identity of this base pair (Table 2). In addition, the rest of the entire P1 stem was mutated where the $5^{\prime}$ and $3^{\prime}$ strand sequences were interconverted (P1 reverse) and a $K_{\mathrm{D}}{ }^{\text {rel }}$ close to unity was obtained. These results show that the nucleotide identity of the P1 stem is relatively unimportant for ligand binding, and that the sequence is most probably conserved to form the alternative "off" secondary structure, which contains the intrinsic transcription terminator (Fig. 1). In addition, the influence of the stability of the P1 stem on 2AP binding was also studied using a series of aptamers having various P1 stem lengths. Of all the aptamers studied, VV1 is the aptamer having the longest P1 stem (9 bp) (Serganov et al. 2004), and was thus used in the following experiments. A series of variants in which the P1 stem varied in length from 2 to $6 \mathrm{bp}$ was generated (Fig. 3C). The 2AP binding affinity for each variant was assessed and a correlation between the length of P1 and the 2AP binding affinity was observed. An aptamer having a P1 stem of only 2 bp shows very little binding affinity compared to the VV1 wild-type variant $\left(K_{\mathrm{D}}{ }^{\text {rel }}=54\right)$. However, the elongation of the P1 stem provides better 2AP binding affinities (Fig. 3C) where 4 bp and 6 bp P1 stem-containing variants exhibit relative affinities of 3.7 and 2.0, respectively. Thus, these results show the importance of the P1 stem length for 2AP binding and indicate that an aptamer having a stem of only $2 \mathrm{bp}$ is able to bind 2AP, 
TABLE 1. Adenine riboswitch aptamers affinity for $2 A P$

\begin{tabular}{lcc}
\hline Aptamer & $K_{\text {Dapp }}(\mathrm{nM})$ & $K_{\mathrm{D}}{ }^{\text {rel }}$ \\
\hline pbuE variant & $354 \pm 17^{\mathrm{a}}$ & $(1.0)$ \\
CP4-add & $552 \pm 36$ & 1.6 \\
CPS1-add & $391 \pm 52$ & 1.1 \\
PP1-add & $204 \pm 23$ & 0.6 \\
VP1-add & $177 \pm 28$ & 0.5 \\
VV1-add & $116 \pm 14$ & 0.3 \\
xpt-pbuX G box & $3747 \pm 288$ & 11 \\
C65U & $390 \pm 31$ & 1.1 \\
\hline
\end{tabular}

${ }^{a}$ Determined in a previous study. Apparent dissociation constants $\left(K_{\text {Dapp }}\right)$ were measured under standard conditions for $\mathrm{A}$ box variants used in these studies. $K_{\mathrm{D}}{ }^{\text {rel }}$ is the factor by which a given variant is impaired relative to the natural pbuE A box variant, i.e., $K_{\text {Dapp }}\left(\right.$ variant) $/ K_{\text {Dapp }}$ (pbuE variant).

albeit to a weaker level when compared to the wild-type molecule.

\section{Effect of position 15 on ligand binding}

In the crystal structure of the adenine aptamer, the adenine at position 15 is involved in an AA platform from which A15 intercalates between G63 and A64 (Serganov et al. 2004). This nucleotide at position 15 is strictly conserved according to our alignment (Fig. 2A), and has also been noted in a recent review describing the adenine aptamer consensus secondary structure (Breaker 2004). Mutations were introduced at position 15 , and the $2 \mathrm{AP}$ binding affinity of the resulting variants was assessed (Table 2). The presence of a guanine at this position leads to a $2 \mathrm{AP}$ binding affinity that is reduced fivefold, while introducing a uracil further decreases affinity $\left(K_{\mathrm{D}}{ }^{\text {rel }}=8.4\right)$. However, $2 \mathrm{AP}$ binding is drastically impaired in an A box aptamer having an A15C mutation (Fig. 4A). These results suggest that the identity of nucleotide 15 is important for the A box aptamer to bind $2 \mathrm{AP}$ and a summary of the results is provided (Fig. 4B).

The drastic 2AP binding affinity reduction observed for the $\mathrm{A} 15 \mathrm{C}$ variant prompted us to investigate if the looploop interaction is compromised in the presence of this mutation. Indeed, crystal structures have shown that a loop-loop interaction occurs between the P2 and the P3 loops (Batey et al. 2004; Serganov et al. 2004; Gilbert et al. 2006). This tertiary interaction is important for ligand binding (Lemay et al. 2006), and replacement of the wildtype loops with stable tetraloops abolishes the ability of the aptamer to recognize hypoxanthine (Batey et al. 2004). Therefore, we sought for evidences relating weak $2 \mathrm{AP}$ affinity and severe disruption of the loop-loop interaction by using a native gel electrophoresis assay. This technique can be used to study global conformational changes in nucleic acids whereby a compact structure generally migrates faster than a more extended one (Lilley 1998;
Silverman and Cech 1999; Silverman et al. 1999; Yamauchi et al. 2005). We reasoned that aptamers having a functional loop-loop interaction should have a compact structure when compared to variants compromised in the interaction, and should therefore display a faster migration rate. A control was made in which the sequence of the P2 loop was changed for its complementary sequence (P2 comp variant), resulting in an aptamer in which the formation of the loop-loop structure is compromised. The migration was performed in the presence of $1 \mathrm{mM}$ magnesium ions, and shows retarded mobility for the P2 comp variant compared to the wild-type molecule, confirming its inability to form the loop-loop interaction (Fig. 4C). However, as for other variants, the $\mathrm{A} 15 \mathrm{C}$ molecule exhibits a migration similar to the wild type, suggesting that the

TABLE 2. Effect of sequence changes in adenine aptamers

\begin{tabular}{|c|c|c|}
\hline Aptamer & $K_{\text {Dapp }}(\mathrm{nM})$ & $K_{\mathrm{D}}{ }^{\text {rel }}$ \\
\hline \multicolumn{3}{|l|}{ A. $A$ box } \\
\hline A12U-U66A & $1334 \pm 128$ & 3.8 \\
\hline A20U-U32A & $308 \pm 18$ & 0.9 \\
\hline A46U-U62A & $1231 \pm 117$ & 3.5 \\
\hline C45G-G63C & $2889 \pm 219$ & 8.2 \\
\hline A49U-U59A & $417 \pm 27$ & 1.2 \\
\hline P1 reverse ${ }^{a}$ & $342 \pm 13$ & 1.0 \\
\hline \multicolumn{3}{|l|}{ B. P1 length ${ }^{b}$} \\
\hline P1-2 bp & $6231 \pm 1168$ & $54^{c}$ \\
\hline P1-4 bp & $427 \pm 51$ & $3.7^{\mathrm{c}}$ \\
\hline P1-6 bp & $233 \pm 31$ & $2.0^{\mathrm{c}}$ \\
\hline \multicolumn{3}{|l|}{ C. Positions 15 and 39} \\
\hline A15C & N.D. ${ }^{d}$ & N.D. ${ }^{d}$ \\
\hline A15G & $1946 \pm 186$ & 5.5 \\
\hline A15U & $2991 \pm 335$ & 8.4 \\
\hline G39A & $1977 \pm 139$ & 5.6 \\
\hline G39C & $341 \pm 23$ & 1.0 \\
\hline G39U & $210 \pm 18$ & 1.7 \\
\hline A box U65C & N.D. ${ }^{d}$ & N.D. ${ }^{d}$ \\
\hline A box U65C-G39U & $4124 \pm 399$ & 11 \\
\hline G box U39G & N.D. ${ }^{d}$ & N.D. ${ }^{d}$ \\
\hline VV1 U39A & $1591 \pm 217$ & $14^{\mathrm{c}}$ \\
\hline
\end{tabular}

Apparent dissociation constants $\left(K_{\text {Dapp }}\right)$ were measured under standard conditions for A box variants used in these studies (see Materials and Methods). $K_{\mathrm{D}}{ }^{\text {rel }}$ is the factor by which a given variant is impaired relative to the natural pbuE A box variant, i.e., $K_{\text {Dapp }}$ (variant) $/ K_{\text {Dapp }}$ (natural).

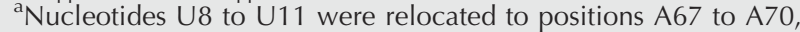
and positions $A 67$ to $A 79$ were relocated to positions U8 to U11. 'The following $5^{\prime}$ side of the P1 stem sequences are shown for the various lengths (uppercase letters indicate nucleotides involved in the formation of the P1 stem while lowercase represents singlestranded nucleotides (all written $5^{\prime}$ to $3^{\prime}$ ): $2 \mathrm{bp}$, gcgUA; $4 \mathrm{bp}$, gcgCAUA; 6 bp, gcgUUCAUA.

${ }^{c} K_{D}{ }^{\text {rel }}$ were reported as a function of the obtained $K_{\text {Dapp }}$ for the VV1 wild-type aptamer and were calculated as the ratio of $K_{\text {Dapp }}$ (variant) $/ K_{\text {Dapp }}(V V 1)$.

${ }^{\mathrm{d}}$ The low 2AP fluorescence quenching due to inefficient binding makes calculation of dissociation constant unreliable (e.g., $K_{\text {Dapp }}>$ $25 \mu \mathrm{M})$ and is not determined (N.D.). 
A

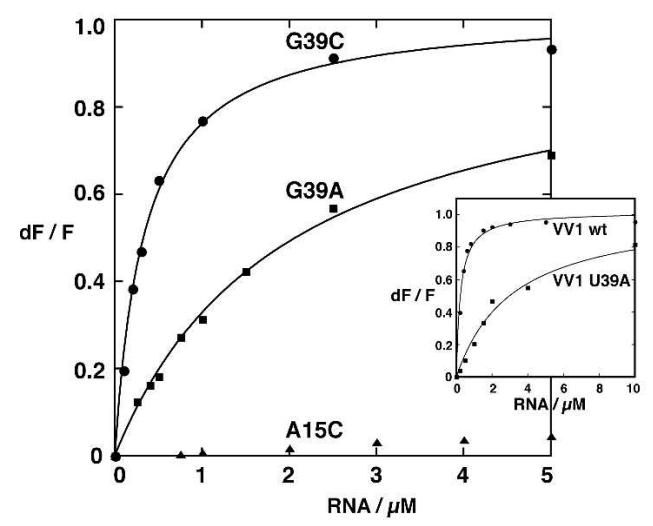

B

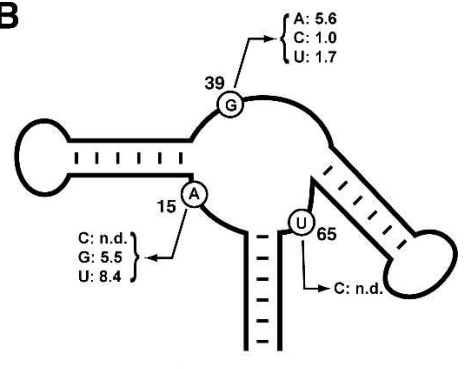

Adenine aptamer

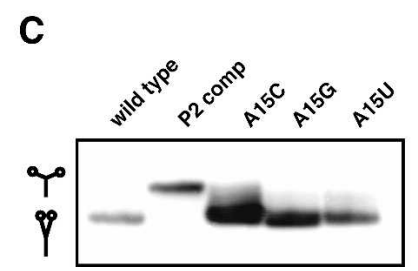

D

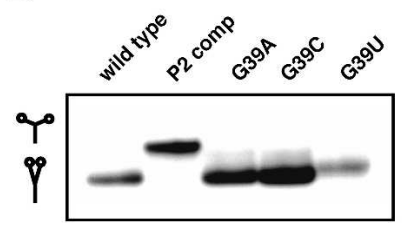

FIGURE 4. The importance of nucleotide positions 15 and 39 for $2 \mathrm{AP}$ binding. (A) Normalized 2AP fluorescence intensities as a function of RNA concentration for G39A (squares), G39C (circles), and $\mathrm{A} 15 \mathrm{C}$ (triangles). The inset shows the influence of an adenine at position 39 in the context of the VV1 aptamer. The best fit is not reported for A15C, as the very weak fluorescence quenching observed precludes a reliable estimation of the fitting parameters (e.g., $R$ value). $(B)$ Schematic representing the different $2 \mathrm{AP}$ binding affinity reductions observed for positions 15,39 , and 65 . Note that the $2 \mathrm{AP}$ binding affinities of the $\mathrm{A} 15 \mathrm{C}$ and $\mathrm{U} 65 \mathrm{C}$ variants could not be determined due to their very low affinity. $(C)$ Effect of A15 substitutions on loop-loop formation. Nondenaturing gel electrophoresis of the natural (wt), P2 comp, and variants mutated at position 15 in the presence of $1 \mathrm{mM}$ magnesium ions. The control loop variant P2 comp exhibits a retarded electrophoretic migration rate. (D) Effect of G39 substitutions on the loop-loop formation. Nondenaturing gel electrophoresis of the natural (wt), P2 comp, and variants mutated at position 39 in the presence of $1 \mathrm{mM}$ magnesium ions. The control loop variant P2 comp exhibits a retarded electrophoretic migration rate.

loop-loop interaction is formed. The presence of the looploop interaction of the $\mathrm{A} 15 \mathrm{C}$ variant was also independently confirmed by performing a partial RNase T1 assay (data not shown).

\section{Effect of position 39 on ligand binding}

Upon examination of the crystal structure, it can be observed that nucleotide 39 is completely exposed to the solvent (Serganov et al. 2004). Based on our consensus structure presented in Figure 2, the identity of position 39 exhibits the B nucleotide consensus ( $\mathrm{C}, \mathrm{G}$, or $\mathrm{U})$. To test if this consensus stems from a structural constraint or from our relatively small number of sequences, pbuE aptamer variants were engineered by introducing mutations at position 39. The presence of a cytosine does not significantly affect $2 \mathrm{AP}$ binding affinity (Fig. 4A), while a uracil slightly increases the ligand affinity by a factor of 1.7 . However, introduction of an adenine at position 39 significantly lowers the binding affinity by a factor of 5.6-fold (Fig. 4A). A similar mutation (e.g., U39A) was introduced in the context of the VV1 aptamer and a decrease of 14 -fold was obtained when compared to the VV1 wildtype molecule (Fig. 4A, inset). Thus, compared to other nucleotide substitutions, the introduction of an adenine at position 39 has an adverse effect on 2AP binding, and this provides a functional basis for the $B$ nucleotide consensus derived from our sequence alignment (Fig. 2A). The loop-loop interaction was also studied in all $p b u E$ variants, and it was found that the interaction is not compromised when position 39 is mutated (Fig. 4D).

\section{Functional correlation between positions 39 and 65}

The phylogenetic and functional requirements for the lack of an adenine at position 39 in the A box aptamer lead us to hypothesize that this restriction could be related to the ligand specificity of the aptamer. Thus, for the A box aptamer, an adenine at position 39 has a negative effect on ligand binding when a uracil is present at position 65. Furthermore, this hypothesis suggests that the identity of position 39 should have a different requirement in the context of the guanine riboswitch. Indeed, examination of 29 guanine riboswitch aptamer sequences revealed that the identity of the nucleotide at position 39 exhibits the $\mathrm{H}$ nucleotide consensus (A, C, and $\mathrm{U}$ ) (Mandal et al. 2003). To establish whether the presence of a guanine at position 39 would be detrimental for ligand binding, the $x p t-p b u X$ guanine-specific aptamer was used in which a guanine was introduced at position 39 (Fig. 5A). The 2AP binding affinity of the resulting aptamer is strongly reduced when compared to the natural $x p t-p b u X$ guanine aptamer (cf. Figs. 3B and 5A). This result suggests that the identity of position 39 is also important in the context of the G box. Thus, nucleotides introduced at position 39 giving the weakest 2AP binding variants in the context of the adenine and guanine aptamers are an adenine and a guanine, respectively, indicating that a combination of nucleotides A39-U65 or G39-C65 is reducing 2AP affinity.

Crystal structures (Batey et al. 2004; Serganov et al. 2004) and mutational analysis (Mandal and Breaker 2004a) have 
A
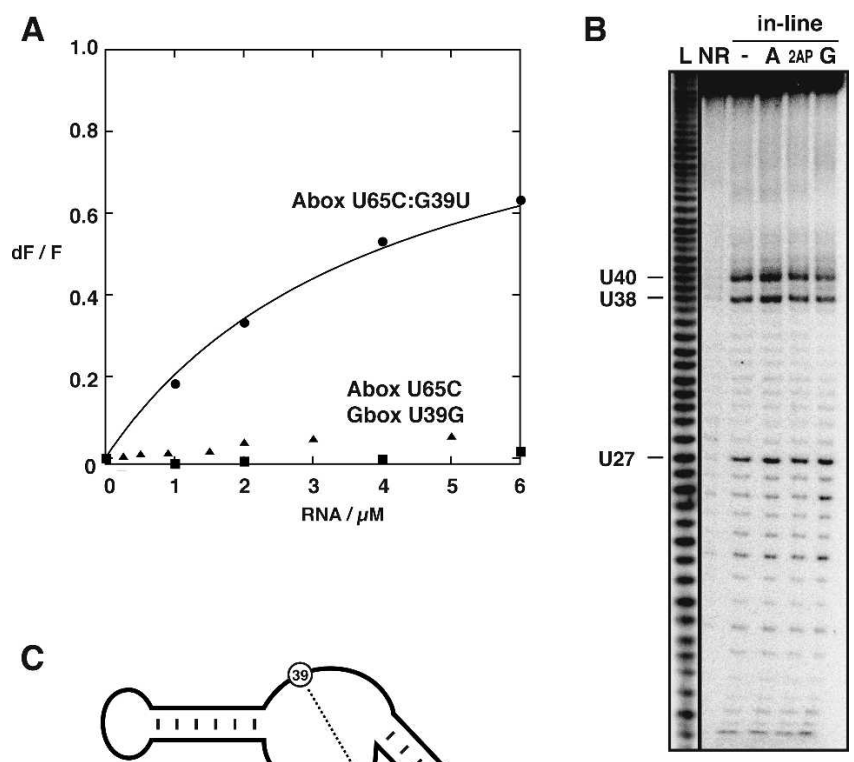

\section{C}

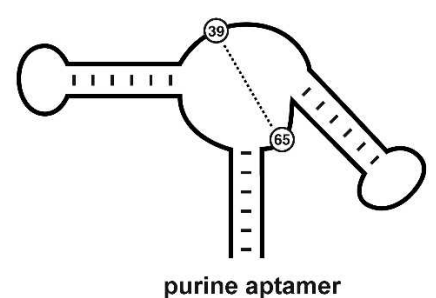

FIGURE 5. Functional correlation between nucleotides 39 and 65 . (A) Normalized 2AP fluorescence intensities as a function of RNA concentration for A box U65C (triangles), G box U39G (squares), and A box G39U-U65C (circles). Best fits are not reported for A box $\mathrm{U} 65 \mathrm{C}$ and G box U39G, as very poor $2 \mathrm{AP}$ fluorescence quenching is detected for these variants. (B) Introduction of a U65C mutation alters the local structure of the $p b u E$ A box aptamer. In-line probing assays in the presence of adenine (A), 2-aminopurine (2AP), or guanine $(\mathrm{G})$. Radioactively $\left[5^{\prime}-{ }^{32} \mathrm{P}\right]$-labeled RNA molecules were incubated for $72 \mathrm{~h}$ at room temperature in the absence of a ligand (-) or in the presence of $10 \mu \mathrm{M}$ ligand to allow spontaneous backbone scissions, and the products were separated by denaturing PAGE. Lanes NR and $L$ : A box RNA molecules that were nonreacted (NR) or partially digested by alkali (L). The major cleavage products are indicated on the left. Note that the ladder is taken from an independent study. $(C)$ Schematic of a purine aptamer indicating a possible interaction between positions 39 and 65 .

shown that the riboswitch specificity is largely determined by the identity of nucleotide 65 , which is involved in base pairing with the ligand. Thus, we speculated that an unproductive interaction restraining the aptamer to bind a ligand could occur between nucleotides 39 and 65. The wild-type $p b u E$ adenine riboswitch aptamer contains a guanine at position 39, and thus its conversion to a guanine-specific aptamer (U65C) should be detrimental for the 2AP binding activity given that a G39-C65 combination is present in this variant. As expected, the $\mathrm{A}$ box U65C variant exhibits a very poor 2AP binding affinity (Fig. 5A). However, to ascertain that it is not the introduction of a cytosine at position 65 in the context of the A box that inhibits 2AP binding, the guanine at position 39 was mutated for a uracil (G39U), and a $K_{\mathrm{D}}{ }^{\text {rel }}$ of 11 was obtained yielding an affinity identical to the wild-type guanine aptamer (Table 1). This result suggests that reduction of 2AP binding is probably caused by an interaction between nucleotides 39 and 65, which makes the aptamer inefficient for ligand binding.

The A box U65C variant was further characterized using an in-line probing assay. This assay exploits the inherent chemical instability of RNA under physiological conditions that are primarily due to the cleavage of phosphodiester linkages (Soukup and Breaker 1999). Spontaneous cleavage is more pronounced in unstructured single-stranded regions because internucleotide linkages are free to adopt an in-line conformation that is precluded in the context of an A-form helix (Soukup and Breaker 1999). This assay has been extensively used to monitor conformational changes induced by external ligands on numerous mRNA leader regions (Nahvi et al. 2002; Winkler et al. 2002a,b, 2004; Mandal et al. 2003, 2004; Winkler and Breaker 2003; Mandal and Breaker 2004a). The wild-type pbuE adenine riboswitch aptamer has been previously studied using the in-line probing technique where it was found that the core of the aptamer is more constrained in the presence of adenine and 2AP (Mandal and Breaker 2004a). We examined the pattern of spontaneous cleavage of RNA for the A box U65C in the absence of ligand, and in the presence of adenine, 2AP, and guanine (Fig. 5B). In the absence of any ligand, cleavage products that correspond to positions U27, U38, and U40 are obtained. This pattern slightly differs from those obtained for both A and G box wild-type molecules (Mandal et al. 2003; Mandal and Breaker 2004a). In both cases, the core of the aptamer is cleaved at more sites than observed for the A box U65C variant. Moreover, both A and G box wild-type aptamers exhibit cleavages at position 65 in contrast to what we obtain here with the A box U65C, indicating that it is more constrained in the context of a U65C mutation. The addition of adenine or 2AP does not alter the pattern of spontaneous cleavage products, indicating that $2 \mathrm{AP}$ or adenine shows very weak binding affinity (Fig. 5B), therefore supporting our 2AP fluorescence binding assays. Notably, the addition of guanine does not promote a significant reduction at positions U38 and U40, indicating that, in our experimental conditions, the aptamer is not able to bind the ligand efficiently, but not as a result of a disrupted loop-loop interaction as confirmed by native gel assays (data not shown).

\section{DISCUSSION}

Molecular recognition involving the A box motif is particularly interesting because RNA-ligand interactions are potentially very important in the elaboration of antimicrobial agents targeting riboswitches (Winkler and Breaker 2005). Specifically, drug compounds competing with metabolite binding could be used to alter the expression of essential metabolic genes of bacterial pathogens. Clear 
examples of this have been found in the context of lysine (Sudarsan et al. 2003b), thiamine pyrophosphate (Sudarsan et al. 2005), and adenine (Johansen et al. 2003) riboswitches. Previously, two consensus secondary structures have been reported for the A box (Breaker 2004; Soukup and Soukup 2004), but none were tested in an experimental framework. A novel consensus secondary structure describing the A box motif is proposed in this work, which is based on a comparative sequence and functional analysis using six natural variants (Fig. 2). This novel consensus sequence structure has been experimentally tested by a combination of 2AP fluorescence, in-line probing, and native gel electrophoresis assays. The newly proposed consensus structure is similar to previous ones but exhibits differences in helical regions and also in the core of the aptamer. Among those observed in the core, the identity of position 39 appears to be related to the riboswitch specificity, which is largely determined by the identity of position 65 .

All adenine aptamers used in the alignment were studied using $2 \mathrm{AP}$ as a fluorescent ligand (Table 1). Given that sequence variations are found between aptamer sequences, it is expected that minor variations in 2AP binding will be observed. Accordingly, 2AP binding affinities are found to range from low nanomolar to midnanomolar. These values are in excellent agreement with previous work where the adenine and 2AP affinities for the pbuE aptamer were both found to be $300 \mathrm{nM}$ using in-line probing (Mandal and Breaker 2004a). Moreover, examination of the 2AP binding affinity of the $\mathrm{G}$ box aptamer revealed an affinity decrease of 11 -fold compared to the A box $\left(K_{\text {Dapp }} \sim 3.7 \mu \mathrm{M}\right)$. This value is in good agreement with a previous study where a $K_{\text {Dapp }}$ of $\sim 10 \mu \mathrm{M}$ was estimated using in-line probing (Mandal et al. 2003). The 2AP binding affinity difference observed between the A and $\mathrm{G}$ box motifs could result from the numerous nucleotide variations that exists between both aptamers. However, introduction of a single C65U mutation in the $G$ box aptamer confers a binding affinity very close to the A box (Fig. 3B), showing that their difference in 2AP affinity is most probably caused solely by the identity of the nucleotide at position 65. These results show that 2AP binding assays are complementary to other biochemical methods (e.g., in-line probing) in studying both adenine and the guanine riboswitch aptamers. Indeed, 2AP has recently been used to determine the kinetics of ligand binding corresponding to the $p b u E$ A box motif, where it was suggested that this riboswitch could operate under kinetic control when subjected to certain conditions, but could also switch between kinetic and equilibrium controls in other conditions (Wickiser et al. 2005).

The A box motif is mostly conserved in the core region, but some peripheral helical segments, like P1 and the P3 stems, are also conserved to some extent (Fig. 2B; Mandal and Breaker 2004a). 2AP fluorescence data have shown that the identity of the two P3 junction-proximal base pairs,
C45-G63 and A46-U62, is important for ligand binding (Table 2). Although these base pairs are not directly involved in tertiary interactions in the crystal structure (Serganov et al. 2004), they could be involved in the global architecture of the aptamer given their close proximity to the junction. Indeed, the global architecture of RNA junctions has been shown to be influenced by proximal base pairs by means of coaxial stacking (Lilley 1998). However, native gel and RNAse T1 assays performed on the aptamer variant $\mathrm{C} 45 \mathrm{G}-\mathrm{G} 43 \mathrm{C}$ revealed that the looploop interaction can take place, suggesting that this mutation does not significantly perturb the loop-loop interaction (data not shown). Moreover, 2AP binding data obtained from aptamer variants having P1 stem mutations indicate that reversal of the junction-proximal base pair A12-U66 infers a 3.8-fold decrease in ligand binding. This loss of affinity can readily be explained by the A boxadenine complex crystal structure where the A12-U66 base pair is involved in a base triple, C41•(U66-A12). In this triple, C41 is positioned in the minor groove of the U66-A12 base pair, and this forms a platform for the stacking of another base triple that is involved in the binding of the ligand (Serganov et al. 2004). The reversal of U66-A12 most probably has an adverse effect on the formation of the base triple, which in turn, would negatively influence the formation of the ligand binding site. On the other hand, the adjacent A67-U11 base pair, which is also involved in a base triple, $\mathrm{U} 40 \cdot(\mathrm{A} 67-\mathrm{U} 11)$, seems less important for $2 \mathrm{AP}$ binding given that reversal of this base pair, which is included in the $\mathrm{P} 1$ reverse mutant, yields an aptamer that closely matches the 2AP binding affinity of the wild-type molecule (Table 2). Moreover, the wild-type 2AP binding affinity displayed by the $\mathrm{P} 1$ reverse mutant shows that most of the P1 stem is not conserved for ligand binding. This suggests that the identity of the P1 helix is most probably conserved for its dual role in the formation of the aptamer and in the terminator stem-loop (Fig. 1). However, the presence of the $\mathrm{P} 1$ stem itself is important for ligand binding, as decreasing its length to 2 bp markedly decreases the ligand affinity by a factor of 54-fold (Fig. 3C). This result suggests that although 2AP binding affinity is decreased, A box molecules having a P1 stem of only 2 bp are still able to perform ligand binding. It is expected that the P1 stem stability of these aptamers will be decreased, and the observed low 2AP binding most probably also results from this instability.

As described in a recent review (Breaker 2004), the A box displays a strong evolutionary conservation for A15 (Fig. 2B). While the 2AP fluorescence data show that substitution by a guanine or a uracil decreases the affinity by 5.5 - and 8.4-fold, respectively, the presence of a cytosine markedly reduces the affinity (Fig. 4B). These results indicate that the evolutionary conservation of position 15 is important for ligand binding. The very poor $2 \mathrm{AP}$ binding activity of the A15C mutant is rather intriguing. One explanation for 
the low affinity is that a cytosine at position 15 could interact with G37 thereby preventing it from forming a Watson-Crick base pair with C44 as seen in the crystal structure. The interaction between 15 and 37 could cause a major structural perturbation like the disruption of the loop-loop interaction, which is known to be very important for ligand binding (Lemay et al. 2006). However, none of the mutations introduced in position 15 disrupt the loop-loop interaction, as observed by native gel assays (Fig. 4C).

In addition, the $\mathrm{A}$ box consensus secondary structure presented in Figure $2 \mathrm{~B}$ indicates that position 39 of the aptamer can be any base but not an adenine. This consensus is supported by our 2AP binding results where variants G39C and G39U exhibit 2AP binding affinity near the wild-type level while the introduction of an adenine at position 39 results in a 5.6-fold decrease of 2AP affinity (Table 2). The negative effect of introducing an adenine at this position is emphasized in the context of the VV1 aptamer where a 14-fold decrease of affinity is observed. This reduction does not result from a disruption in the loop-loop interaction as determined by native gel assays (Fig. 4D). Interestingly, the crystal structure indicates that nucleotide 39 is exposed to the solvent as it loops out of the core scaffold (Serganov et al. 2004), and thus is in apparent contradiction with the phylogenic evolutionary requirements and our 2AP binding data. However, the crystal structure does not offer information about the folding pathway of the aptamer, which could be altered in the presence of the G39A variant molecule. Very recently, a mechanism for ligand recognition by the guanine riboswitch has been proposed where nucleotide 39 becomes exposed when the ligand is bound by the aptamer (Gilbert et al. 2006). This is in very good agreement with our results given that this implies nucleotide 39 is not exposed to the solvent in the absence of the ligand, and could therefore interact with surrounding nucleotides in the core of the aptamer, such as nucleotide 65 .

Interestingly, the absence of guanine at this position in the context of the guanine riboswitch aptamer for 29 sequences (Mandal et al. 2003) strongly suggests that a similar consensus sequence constraint is present in the $\mathrm{G}$ box. A series of natural guanine aptamers having the three possible nucleotides at position 39 were tested for $2 \mathrm{AP}$ binding. All of them display affinities close to the $x p t-p b u X$ $\mathrm{G}$ box variant, but all have a significant reduction in $2 \mathrm{AP}$ binding affinity when position 39 is mutated for a guanine (J. Mulhbacher and D.A. Lafontaine, unpubl.). The requirement, for position 39 not to be an adenine in the A box and a guanine in the G box, suggests that the identity of this nucleotide is related to the specificity of the aptamer. In other words, an aptamer having the combination A39-U65 or G39-C65 is not evolutionary favored, and displays reduced ability to bind a ligand. This relationship is further supported by converting the $p b u E$ A box variant (G39) to a guanine-specific aptamer (U65C), which gives very low 2AP binding (Fig. 5A). However, introduction of the G39U mutation (A box G39U:U65C) in the latter results in a rescue of $2 \mathrm{AP}$ binding, as expected.

The A box U65C variant shows a different in-line probing pattern compared to the wild-type molecules of the A box or G box aptamers (Fig. 5B) (with in-line probing results from Mandal et al. 2003; Mandal and Breaker 2004a; Lemay et al. 2006). This suggests that the unbound variant has a structure different from both wildtype molecules, and provides a structural explanation for the observed decrease in 2AP binding affinity. Similar conclusions were obtained in a recent study showing that the affinity of the A box U65C variant for guanine was decreased by a factor of at least 10-fold (Mandal and Breaker 2004a). It was also found that a $\sim 100$-fold derepression in $\beta$-galactosidase activity was observed when a single U65C mutation was introduced in a pbuE adenine riboswitch-lacZ fusion construct. The derepression was attributed to the destabilization of the terminator helix that was caused by the introduction of the U65C mutation. An additional factor to the derepression observed in vivo could be that the U65C mutation, in the context of the $p b u E$ variant that contains a guanine at position 39 , allows the aptamer to stabilize the P1 stem without the presence of the ligand, thereby shifting the riboswitch conformers' equilibrium toward the "on" structure. Inspection of the conformation and position of residues U39 in the crystal structure of the A box riboswitch aptamer (1Y26) reveals that it is not inconceivable that the base of U39 could, with a small reorganization of the loop region surrounding it, be inserted into the adenine binding site, and if mutated to an adenosine, could form Watson-Crick-type hydrogen bonds with U65, thus taking the place of the adenine substrate. The situation is indeed similar in the G box aptamer (1Y27) where U39 could, if mutated to a guanosine residue, possibly take the place of the guanine substrate and form hydrogen bonds to C65. Most of the other hydrogen bonds and stacking interactions could be usurped by these internal substitutions of mutated residue 39 into the binding site. Some small degree of structural rearrangement would be required, and these are currently being investigated.

The secondary and tertiary structures of guanine- and adenine-specific aptamers are highly similar, and experimental evidence indicate that the interconversion between both can be done by a single mutation introduced at position 65 in the core of the aptamer (Mandal and Breaker 2004a; Serganov et al. 2004). This suggests that such changes might occur with high frequency in natural populations. However, neither a single-base variant of the $x p t$ or $p b u E$ riboswitches exhibits wild-type affinity in genetic control in vivo, suggesting that multiple mutations are involved in ligand recognition (Mandal and Breaker 2004a). Position 39 seems to be particularly important for ligand specificity in the context of the guanine riboswitch. 
Thus, accessory mutations that do not directly define ligand specificity, but that further adjust binding affinity, might be very important for purine riboswitches in a biological environment.

\section{MATERIALS AND METHODS}

\section{Purification of DNA oligonucleotides}

Oligonucleotides were purchased from Sigma Genosys (Canada). Oligonucleotides were purified by denaturing polyacrylamide gel electrophoresis, electroeluted into $8 \mathrm{M}$ ammonium acetate, recovered by ethanol precipitation and dissolved in water.

\section{Synthesis of RNA molecules}

RNA was transcribed from a double-stranded DNA template using T7 RNA polymerase (Milligan et al. 1987). Templates for aptamers were made from DNA oligonucleotides (200 pmol) containing the aptamer sequence and the T7 promoter sequence that were incubated in a $200 \mu \mathrm{L}$ mixture containing $10 \mathrm{mM}$ Tris$\mathrm{HCl}, \mathrm{pH} 7.5,10 \mathrm{mM} \mathrm{MgCl}_{2}$, and $50 \mathrm{mM} \mathrm{KCl}$, denatured by heating at $95^{\circ} \mathrm{C}$ for $1 \mathrm{~min}$, and allowed to cool slowly to $37^{\circ} \mathrm{C}$. The in vitro transcription reactions were carried out for $3 \mathrm{~h}$. RNA was purified by denaturing PAGE and recovered as described for DNA oligonucleotides. All transcribed RNA species begin with a $5^{\prime}$ GCG sequence to minimize the $5^{\prime}$-heterogeneity of the RNA population (Pleiss et al. 1998). The following oligos were used (all sequences written $5^{\prime}$ to $3^{\prime}$ ):

\section{A box: GCGCGUUGUAUAACCUCAAUAAUAUGGUUUGAGG GUGUCUACCAGGAACCGUAAAAUCCUGAUUACAACGC; G box: GCGCACUCAUAUAAUCGCGUGGAUAUGGCACGCA AGUUUCUACCGGGCACCGUAAAUGUCCGACUAUGGG UGCGC.}

Mutants were also prepared with the sequence changes indicated in the text.

\section{Fluorescence spectroscopy}

Prior to 2AP fluorescence measurements, the RNA was heated for $1 \mathrm{~min}$ to $95^{\circ} \mathrm{C}$ in water and then preincubated at $23^{\circ} \mathrm{C}$ for $10 \mathrm{~min}$ in the reaction buffer (see below) to ensure homogeneous folding of RNA species (Perrotta et al. 1999). Fluorescence spectroscopy was performed on a Quanta Master fluorometer. All data were collected at $25^{\circ} \mathrm{C}$ in $10 \mathrm{mM} \mathrm{MgCl}_{2}, 50 \mathrm{mM}$ Tris- $\mathrm{HCl}, \mathrm{pH}$ 8.3, and $100 \mathrm{mM} \mathrm{KCl}$. Spectra were corrected for background, and intensities were determined by integrating the data collected over the range 330-450 $\mathrm{nm}$. Excitation for 2-aminopurine fluorescence was done at $300 \mathrm{~nm}$ to obtain a good separation between the Raman peak and the 2AP-fluorescence signal.

The fraction of quenched 2AP fluorescence was determined by monitoring the fluorescence emission of a fixed concentration of $2 \mathrm{AP}$ $(50 \mathrm{nM})$ and titrating with an increasing concentration of a given aptamer RNA molecule. If the total aptamer concentration is in large excess relative to $2 \mathrm{AP}$, then it can be assumed that the concentration of the free aptamer is similar to the concentration of total aptamer molecules. The binding can thus be described by the equation

$$
d F / F=(1-a)[\mathrm{RNA}] /\left(K_{\mathrm{Dapp}}+[\mathrm{RNA}]\right)
$$

where $d F$ is the change in fluorescence intensity, $F$ is the fluorescence intensity in the absence of RNA, and $K_{\text {Dapp }}$ is the apparent dissociation constant. The parameter $a$ is a dimensionless constant proportional to the ratio of the quantum yields of the 2AP in the complex and free solutions. $a$ is also less than unity as the quantum yield of $2 \mathrm{AP}$ free is higher than in the complex. The $a$ value is obtained, together with $K_{\text {Dapp }}$, by nonlinear leastsquares fitting following the Levenberg-Marquardt algorithm and typically corresponds to a value of 0.05 . The equation assumes a simple 1:1 stoichiometry between the RNA and 2AP, as it has been reported in the various crystal structures of aptamer-ligand complexes (Batey et al. 2004; Serganov et al. 2004). The reported errors are the standard uncertainties of the data from the best-fit theoretical curves. The standard uncertainty of the measurement is thus assumed to be approximated by the standard deviation of the points from the fitted curve (Flannery et al. 1992; Rist and Marino 2001). For each experiment, at least three measurements have been performed and all exhibited very similar uncertainties from best-fit curves.

\section{Gel electrophoresis under nondenaturing conditions}

Radioactively $\left[5^{\prime}-{ }^{32} \mathrm{P}\right]$-labeled A box RNA (final concentration $<1 \mathrm{nM}$ ) in $90 \mathrm{mM}$ Tris and $89 \mathrm{mM}$ boric acid, $\mathrm{pH} 8.5$ (TB buffer), and $1 \mathrm{mM} \mathrm{MgCl}_{2}$ was incubated at $25^{\circ} \mathrm{C}$ for 5 min before loading. RNA was electrophoresed in 10\% acrylamide:bisacrylamide (29:1) gels in $\mathrm{TB}$ with $\mathrm{MgCl}_{2}$ as required at room temperature at $100 \mathrm{~V}$ for $24 \mathrm{~h}$. The running buffer was circulated during electrophoresis. Dried gels were exposed to PhosphorImager screens.

\section{In-line probing of adenine riboswitch aptamers}

Radioactively $\left[5^{\prime}-{ }^{32} \mathrm{P}\right]$-labeled RNA molecules (final concentration $<1 \mathrm{nM}$ ) were incubated for $72 \mathrm{~h}$ at $25^{\circ} \mathrm{C}$ in a buffer containing $50 \mathrm{mM}$ Tris- $\mathrm{HCl}, \mathrm{pH} 8.3,20 \mathrm{mM} \mathrm{MgCl}_{2}$, and $100 \mathrm{mM}$ $\mathrm{KCl}$ in the absence or presence of $10 \mu \mathrm{M}$ ligand. At the end of each incubation, in-line probing cleavage products were separated by electrophoresis in $10 \%$ polyacrylamide gels containing $7 \mathrm{M}$ urea. Dried gels were exposed to PhosphorImager screens.

\section{ACKNOWLEDGMENTS}

We thank members of the Lafontaine laboratory for helpful discussions, and Drs. Carlos Penedo and Alain Lavigueur for critical reading of the manuscript. We also thank Dr. David Norman for discussions on the possible structural implications of residue 39 mutations. This work was supported by the National Sciences and Engineering Research Council of Canada and the Universite de Sherbrooke (start-up grant). D.A.L. is a Chercheur-Boursier Junior I of the Fonds de la Recherche en Santé du Québec.

Received May 11, 2006; accepted November 21, 2006.

\section{REFERENCES}

Altschul, S.F., Gish, W., Miller, W., Myers, E.W., and Lipman, D.J. 1990. Basic local alignment search tool. J. Mol. Biol. 215: 403-410. 
Batey, R.T., Gilbert, S.D., and Montange, R.K. 2004. Structure of a natural guanine-responsive riboswitch complexed with the metabolite hypoxanthine. Nature 432: 411-415.

Bengert, P. and Dandekar, T. 2004. Riboswitch finder-A tool for identification of riboswitch RNAs. Nucleic Acids Res. 32: W154W159.

Breaker, R.R. 2004. Natural and engineered nucleic acids as tools to explore biology. Nature 432: 838-845.

Cromie, M.J., Shi, Y., Latifi, T., and Groisman, E.A. 2006. An RNA sensor for intracellular $\mathrm{Mg}^{2+}$. Cell 125: 71-84.

Edwards, T.E. and Ferre-D'Amare, A.R. 2006. Crystal structures of the thi-box riboswitch bound to thiamine pyrophosphate analogs reveal adaptive RNA-small molecule recognition. Structure 14: 1459-1468.

Epshtein, V., Mironov, A.S., and Nudler, E. 2003. The riboswitchmediated control of sulfur metabolism in bacteria. Proc. Natl. Acad. Sci. 100: 5052-5056.

Flannery, B.P., Teukolsky, S.A., and Vetterling, W.T. 1992. Numerical recipes in Fortran, 2nd ed. Cambridge University Press, Cambridge, UK.

Gilbert, S.D., Stoddard, C.D., Wise, S.J., and Batey, R.T. 2006. Thermodynamic and kinetic characterization of ligand binding to the purine riboswitch aptamer domain. J. Mol. Biol. 359: 754768.

Grundy, F.J., Lehman, S.C., and Henkin, T.M. 2003. The L box regulon: Lysine sensing by leader RNAs of bacterial lysine biosynthesis genes. Proc. Natl. Acad. Sci. 100: 12057-12062.

Johansen, L.E., Nygaard, P., Lassen, C., Agerso, Y., and Saxild, H.H. 2003. Definition of a second Bacillus subtilis pur regulon comprising the pur and xpt-pbuX operons plus pbuG, nupG (yxjA), and pbuE (ydhL). J. Bacteriol. 185: 5200-5209.

Klein, D.J. and Ferre-D'Amare, A.R. 2006. Structural basis of glmS ribozyme activation by glucosamine-6-phosphate. Science 313: $1752-1756$.

Kubodera, T., Watanabe, M., Yoshiuchi, K., Yamashita, N., Nishimura, A., Nakai, S., Gomi, K., and Hanamoto, H. 2003. Thiamine-regulated gene expression of Aspergillus oryzae thiA requires splicing of the intron containing a riboswitch-like domain in the $5^{\prime}$-UTR. FEBS Lett. 555: 516-520.

Lemay, J.F., Penedo, J.C., Tremblay, R., Lilley, D.M., and Lafontaine, D.A. 2006. Folding of the adenine riboswitch. Chem. Biol. 13: 857-868.

Lescoute, A. and Westhof, E. 2005. Riboswitch structures: Purine ligands replace tertiary contacts. Chem. Biol. 12: 10-13.

Lilley, D.M. 1998. Folding of branched RNA species. Biopolymers 48: 101-112.

Mandal, M. and Breaker, R.R. 2004a. Adenine riboswitches and gene activation by disruption of a transcription terminator. Nat. Struct. Mol. Biol. 11: 29-35.

Mandal, M. and Breaker, R.R. 2004b. Gene regulation by riboswitches. Nat. Rev. Mol. Cell Biol. 5: 451-463.

Mandal, M., Boese, B., Barrick, J.E., Winkler, W.C., and Breaker, R.R. 2003. Riboswitches control fundamental biochemical pathways in Bacillus subtilis and other bacteria. Cell 113: $577-586$.

Mandal, M., Lee, M., Barrick, J.E., Weinberg, Z., Emilsson, G.M., Ruzzo, W.L., and Breaker, R.R. 2004. A glycine-dependent riboswitch that uses cooperative binding to control gene expression. Science 306: 275-279.

McDaniel, B.A., Grundy, F.J., Artsimovitch, I., and Henkin, T.M. 2003. Transcription termination control of the $S$ box system: Direct measurement of S-adenosylmethionine by the leader RNA. Proc. Natl. Acad. Sci. 100: 3083-3088.

Milligan, J.F., Groebe, D.R., Witherell, G.W., and Uhlenbeck, O.C. 1987. Oligoribonucleotide synthesis using T7 RNA polymerase and synthetic DNA templates. Nucleic Acids Res. 15: 8783-8798.

Mironov, A.S., Gusarov, I., Rafikov, R., Lopez, L.E., Shatalin, K., Kreneva, R.A., Perumov, D.A., and Nudler, E. 2002. Sensing small molecules by nascent RNA: A mechanism to control transcription in bacteria. Cell 111: 747-756.
Montange, R.K. and Batey, R.T. 2006. Structure of the S-adenosylmethionine riboswitch regulatory mRNA element. Nature 441: $1172-1175$.

Nahvi, A., Sudarsan, N., Ebert, M.S., Zou, X., Brown, K.L., and Breaker, R.R. 2002. Genetic control by a metabolite binding mRNA. Chem. Biol. 9: 1043.

Nahvi, A., Barrick, J.E., and Breaker, R.R. 2004. Coenzyme B12 riboswitches are widespread genetic control elements in prokaryotes. Nucleic Acids Res. 32: 143-150.

Nudler, E. and Mironov, A.S. 2004. The riboswitch control of bacterial metabolism. Trends Biochem. Sci. 29: 11-17.

Nygaard, P. and Saxild, H.H. 2005. The purine efflux pump PbuE in Bacillus subtilis modulates expression of the PurR and G-box (XptR) regulons by adjusting the purine base pool size. J. Bacteriol. 187: 791-794.

Perrotta, A.T., Shih, I., and Been, M.D. 1999. Imidazole rescue of a cytosine mutation in a self-cleaving ribozyme. Science 286: 123 126.

Pleiss, J.A., Derrick, M.L., and Uhlenbeck, O.C. 1998. T7 RNA polymerase produces $5^{\prime}$ end heterogeneity during in vitro transcription from certain templates. RNA 4: 1313-1317.

Rist, M. and Marino, J. 2001. Association of an RNA kissing complex analyzed using 2-aminopurine fluorescence. Nucleic Acids Res. 29: 2401-2408.

Serganov, A., Polonskaia, A., Phan, A.T., Breaker, R.R., and Patel, D.J. 2006. Structural basis for gene regulation by a thiamine pyrophosphate-sensing riboswitch. Nature 441: 1167-1171.

Serganov, A., Yuan, Y.R., Pikovskaya, O., Polonskaia, A., Malinina, L., Phan, A.T., Hobartner, C., Micura, R., Breaker, R.R., and Patel, D.J. 2004. Structural basis for discriminative regulation of gene expression by adenine- and guanine-sensing mRNAs. Chem. Biol. 11: 1729-1741.

Silverman, S.K. and Cech, T.R. 1999. Energetics and cooperativity of tertiary hydrogen bonds in RNA structure. Biochemistry 38: 86918702.

Silverman, S.K., Zheng, M., Wu, M., Tinoco Jr., I., and Cech, T.R. 1999. Quantifying the energetic interplay of RNA tertiary and secondary structure interactions. RNA 5: 1665-1674.

Soukup, G.A. and Breaker, R.R. 1999. Relationship between internucleotide linkage geometry and the stability of RNA. RNA 5: 13081325.

Soukup, J.K. and Soukup, G.A. 2004. Riboswitches exert genetic control through metabolite-induced conformational change. Curr. Opin. Struct. Biol. 14: 344-349.

Sudarsan, N., Barrick, J.E., and Breaker, R.R. 2003a. Metabolitebinding RNA domains are present in the genes of eukaryotes. RNA 9: 644-647.

Sudarsan, N., Wickiser, J.K., Nakamura, S., Ebert, M.S., and Breaker, R.R. 2003b. An mRNA structure in bacteria that controls gene expression by binding lysine. Genes \& Dev. 17: 2688 2697.

Sudarsan, N., Cohen-Chalamish, S., Nakamura, S., Emilsson, G.M., and Breaker, R.R. 2005. Thiamine pyrophosphate riboswitches are targets for the antimicrobial compound pyrithiamine. Chem. Biol. 12: $1325-1335$.

Thore, S., Leibundgut, M., and Ban, N. 2006. Structure of the eukaryotic thiamine pyrophosphate riboswitch with its regulatory ligand. Science 312: 1208-1211.

Tucker, B.J. and Breaker, R.R. 2005. Riboswitches as versatile gene control elements. Curr. Opin. Struct. Biol. 15: 342-348.

Wickiser, J.K., Cheah, M.T., Breaker, R.R., and Crothers, D.M. 2005. The kinetics of ligand binding by an adenine-sensing riboswitch. Biochemistry 44: 13404-13414.

Winkler, W.C. 2005. Riboswitches and the role of noncoding RNAs in bacterial metabolic control. Curr. Opin. Chem. Biol. 9: 594-602.

Winkler, W., Nahvi, A., and Breaker, R.R. 2002a. Thiamine derivatives bind messenger RNAs directly to regulate bacterial gene expression. Nature 419: 952-956. 
Winkler, W.C., Cohen-Chalamish, S., and Breaker, R.R. 2002b. An mRNA structure that controls gene expression by binding FMN. Proc. Natl. Acad. Sci. 99: 15908-15913.

Winkler, W.C. and Breaker, R.R. 2003. Genetic control by metabolitebinding riboswitches. ChemBioChem 4: 1024-1032.

Winkler, W.C. and Breaker, R.R. 2005. Regulation of bacterial gene expression by riboswitches. Annu. Rev. Microbiol. 59: 487-517.

Winkler, W.C., Nahvi, A., Sudarsan, N., Barrick, J.E., and Breaker, R.R. 2003. An mRNA structure that controls gene expression by binding S-adenosylmethionine. Nat. Struct. Biol. 10: 701-707.

Winkler, W.C., Nahvi, A., Roth, A., Collins, J.A., and Breaker, R.R. 2004. Control of gene expression by a natural metabolite-responsive ribozyme. Nature 428: 281-286.

Yamauchi, T., Miyoshi, D., Kubodera, T., Nishimura, A., Nakai, S., and Sugimoto, N. 2005. Roles of $\mathrm{Mg}^{2+}$ in TPP-dependent riboswitch. FEBS Lett. 579: 2583-2588. 

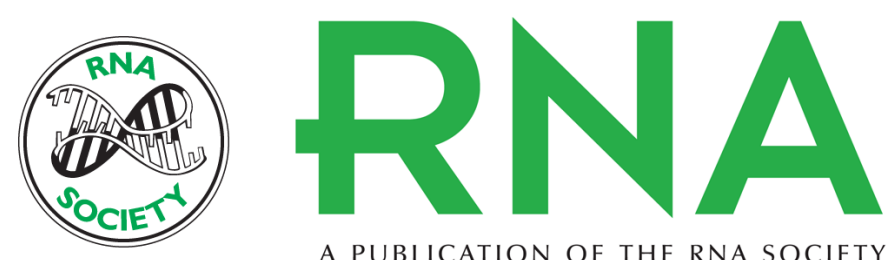

A PUBLICATION OF THE RNA SOCIETY

\section{Core requirements of the adenine riboswitch aptamer for ligand binding}

Jean-FranÇois Lemay and Daniel A. Lafontaine

RNA 2007 13: 339-350 originally published online January 2, 2007

Access the most recent version at doi:10.1261/rna.142007

\section{References This article cites 50 articles, 15 of which can be accessed free at: http://rnajournal.cshlp.org/content/13/3/339.full.html\#ref-list-1}

\section{License}
Email Alerting Receive free email alerts when new articles cite this article - sign up in the box at the Service top right corner of the article or click here.

To subscribe to RNA go to:

http://rnajournal.cshlp.org/subscriptions 